Revue d'histoire de l'Amérique française

REVUE D.HISTOIRE DE L'AMÉRIQUE FRANÇAISE

\title{
Jeunesse de Clio ou la recherche en histoire
}

\section{Claude Galarneau}

Volume 9, numéro 1, juin 1955

URI : https://id.erudit.org/iderudit/301690ar

DOI : https://doi.org/10.7202/301690ar

Aller au sommaire du numéro

Éditeur(s)

Institut d'histoire de l'Amérique française

ISSN

0035-2357 (imprimé)

1492-1383 (numérique)

Découvrir la revue

Citer cet article

Galarneau, C. (1955). Jeunesse de Clio ou la recherche en histoire. Revue

d'histoire de l'Amérique française, 9(1), 3-13. https://doi.org/10.7202/301690ar d'utilisation que vous pouvez consulter en ligne.

https://apropos.erudit.org/fr/usagers/politique-dutilisation/ 


\section{JEUNESSE DE CLIO}

OU

\section{LA RECHERCHE EN HISTOIRE *}

En acceptant de présenter une communication à cet Institut, qui m'accueille aujourd'hui pour la première fois, je me suis rappelé cette phrase de Marc Bloch: "J'aimerais que, parmi les historiens de profession, les jeunes, en particulier, s'habituassent à réfléchir sur ces hésitations, ces perpétuels « repentirs 》 de notre métier. Ce sera pour eux la plus sûre manière de se préparer, par un choix délibéré, à conduire raisonnablement leur effort...» ${ }^{1}$. Je n'ai pas droit au titre d'historien, puisque je n'ai pas encore présenté le chef-d'œuvre artisanal qui ouvre les portes de la corporation, et sans lequel on n'est toujours qu'un répétiteur. Mais je profite de la chance qui m'est offerte pour répondre au désir de Marc Bloch et vous soumettre par la même occasion quelques réflexions sur la recherche en histoire.

Avant tout, je veux rendre hommage à tous ceux qui depuis Garneau, ont maintenu chez nous, vivante, la recherche en histoire, à nos deux universités qui ont créé un enseignement supérieur de l'histoire, et au fondateur ici présent de cet Institut d'Histoire de l'Amérique Française dont la revue est le signe sensible.

Si vous demandez à un honnête homme de chez nous ce qu'il entend en Histoire du Canada, il vous répondra que cela se confond avec la liste des gouverneurs et des évêques, avec les batailles contre les Iroquois et les Anglais, tout au plus avec nos « luttes

* Travail présenté à la réunion générale de l'Institut d'Histoire de l'Amérique française, le 16 avril 1955.

${ }^{1}$ Marc Bloch, Apologie pour l'histoire, Cahiers des Annales, no 3, (Colin, 1949), XVII. 
constitutionnelles ». Si l'on examine sommairement l'histoire que l'on écrivait autrefois, on trouve d'une part des historiens qui ont voulu faire objectif, impartial, qui se sont surtout appliqués consciencieusement à retracer l'histoire des constitutions, des gouvernements, et des luttes parlementaires. D'autre part, des historiens militants qui se donnaient pour mission d'éduquer le peuple, de faire de l'histoire une leçon d'instruction civique et patriotique, puisant à pleines mains dans le trésor des constitutions, des gouvernements et des sessions. On allait même plus loin, on cherchait des arguments pour les combats présents, des justifications pour un engagement politique ou social. C'est ce qui nous a valu de l'histoire apologétique, hagiographique, moralisante. On a pratiqué enfin ce que j'appellerais de l'histoire rapetissante, qui ignore tout de son contexte européen, anglais ou français. - Pourtant, nous ne sommes peut-être pas si retardataires qu'il semble. Car dans bien d'autres pays, les historiens ont très souvent affiché les mêmes préoccupations, ont écrit l'histoire de la même façon, quand leur enseignement universitaire leur aurait permis de faire mieux.

Maintenant que nous comptons trois Instituts d'Histoire, est-ce que tout cela va changer ? Si, il y a bien quelque chose qui changera. Les futurs historiens vont apprendre les rudiments de la méthode critique: recherche et critique des sources, critique externe et critique interne, mise en œuvre des documents, bref toutes les pratiques de l'érudition moderne. Il reste à savoir sur quelle matière s'exercera un si bon instrument de travail. Il n'y a pas de problème de ce côté; on départagera de façon plus certaine patriotes et traîtres, nationalistes et impérialistes. S'il devait en être ainsi, il faudrait se rappeler ce que Valéry a déjà dit de l'histoire: "L'histoire est le produit le plus dangereux que la chimie de l'intellect ait élaboré. Ses propriétés sont bien connues. Il fait rêver, il enivre les peuples, leur engendre de faux souvenirs, exagère leurs réflexes, entretient leurs vieilles plaies, les tourmente dans leur repos, les conduit au délire des grandeurs ou à celui de la persécution, et rend les nations amères, superbes, insupportables et vaines. L'histoire justifie ce que l'on veut. Elle n'enseigne rien, car elle contient tout, et donne des exemples de 
tout. ${ }^{2}$ Cette opinion, qui a toutes les apparences de la vérité, repose pourtant sur une méconnaissance totale de l'histoire. Méconnaissance qui dure toujours et dont la faute retombe, hélas ! en bonne part sur les historiens eux-mêmes. L'histoire n'est pas une boîte à leçons parce que dans ce cas l'histoire est aussi bien, sinon plus, une leçon d'immoralité que de moralité, de vices que de vertus. L'histoire justifie tout ce que l'on veut, Valéry a raison. Rappelons à ceux qui pourraient être tentés de l'oublier que Hitler et les racistes allemands ont cherché la justification de leurs théories et de leurs appétits dans une histoire arrangée selon leurs fins. Pensons à l'usage que font les Soviets de l'histoire. - Mais qu'est-ce donc que l'histoire ?

L'histoire, a-t-on accoutumé de dire, c'est le récit et l'explication du passé. Le passé ce sont les événements, les faits. Et la connaissance de ces événements repose sur les documents, sur des textes. Soumise à tel point que l'histoire devient presque la science des documents. C'est la méthode critique qui triomphe, qui lie l'historien au texte, puisque les faits n'existent pas en dehors des textes. Toute idée directrice, toute idée préconçue au départ étant même interdite: on s'enferme dans les textes et ce sont eux qui nous guident. La connaissance acquise, l'explication suit de façon rigoureuse, logique, rationnelle. Puis le récit se compose, aussi serré, aussi rigoureux. C'est là, très grossièrement, la conception dite scientifique de l'histoire, celle des Langlois et Seignobos ${ }^{3}$. Et certes depuis cinquante ans, l'histoire a accusé des progrès non équivoques. Existe-t-il pour autant une science historique objective, contraignante ${ }^{4}$ ? C'est plus que douteux.

Mais d'abord reprenons cette question de l'objet de l'histoire. L'identification de l'histoire avec le passé et le fait est équivoque, pour ne pas dire plus. Parce que le passé n'est pas un objet de connaissance comme tel ${ }^{5}$, tout le passé de l'humanité étant en

2 Paul Valéry, Regards sur le monde actuel et autres essais (Paris, Gallimard, 1945), 43.

${ }^{3}$ Langlois et Seignobos, Introduction aux études historiques (Paris, Hachette, 1897).

4 Marrou, cité in Hours, Valeur de l'histoire, 52.

$\checkmark$ Bloch, A pologie pour l'histoire, 2. 
cause, on choisit arbitrairement telle série de faits passés contre telle autre. Et qu'est-ce qu'un fait ? Un fait, c'est un événement dont les textes nous ont conservé le souvenir, et que l'on considère comme vrai et important au préalable. Ainsi un fait c'est un événement et un événement c'est un fait. Nous voilà bien avancés.

L'objet de l'histoire, ce n'est pas le passé «ut sic», mais bien l'homme. Etude de l'homme ? entendons-nous bien. Il ne s'agit pas de l'homme en tant qu'individu abstrait mais des hommes en tant que groupes organisés, en tant que sociétés ${ }^{6}$. Et quand nous avons dit l'étude des hommes en tant que groupes, les hommes d'autrefois, nous postulons tout de suite deux notions: celle de temps et d'espace. Les hommes dans le temps et l'espace, donc des hommes qui ont vécu à un moment donné, dans un pays donné. Précisons ces notions de temps et d'espace. De quel temps s'agit-il ? Du temps qui est fait d'une antithèse: le temps qui est d'une part continu, qui ne s'arrête pas, qui se déroule inexorablement, et le temps qui est d'autre part changement, perpétuel changement. On nous a dit maintes fois que l'histoire ne se «répétait » jamais deux fois de la même façon. C'est la seconde partie de l'antithèse. Ces deux aspects, aspect de continuité, aspect de changement sont pourtant liés. Et les grands problèmes, les problèmes fondamentaux de la recherche historique viennent de cette antithèse. C'est tout le problème du permanent et du contingent. - Science des hommes dans l'espace, voilà qu'il faut faire appel à la géographie. Pas à la géographie de nomenclature, mais à celle de Vidal de La Blache et à celle de l'Ecole française contemporaine. - Les hommes ont vécu, ont lutté pour la vie dans un pays donné. Et la connaissance des lieux, du terrain de quelque nature qu'il soit, - terrain physique, économique, linguistique, ethnologique - est essentielle à l'histoire. Histoire et géographie ne se séparent pas. Comment comprendre, par exemple, le paysage rural de la province de Québec, son "géométrisme », si l'on ne se pose le problème des premiers colons français en face de la géographie physique et de l'occupation de la terre?

${ }^{6}$ Bloch, Apologie pour l'histoire, 4. 
Après ce déblaiement initial, il devient plus engageant de parler de certains autres aspects de l'histoire. Nous savons tous que les sciences évoluent rapidement. Nous savons que la physique de 1900 est dépassée, qu'il en est de même de la chimie, des mathématiques. Pourquoi en serait-il autrement des sciences humaines ? Pourquoi persisterait-on à construire en histoire de la même façon qu'en 1900 ? C'est illogique. La connaissance de l'histoire avait été organisée au dix-neuvième siècle en fonction des préoccupations du moment. C'est ce qui nous a valu de l'histoire politique, diplomatique, parlementaire surtout. C'étaient parmi les préoccupations des hommes celles qui, à tort ou à raison, apparaissaient comme les plus importantes chez le plus grand nombre. Mais aujourd'hui, dans la seconde partie du vingtième siècle, il faut organiser notre connaissance de l'histoire en fonction de nos besoins, en fonction de la vie actuelle. C'est là, il me semble, l'orientation à donner à nos recherches.

Or, quels sont nos besoins ? ou plutôt quels sont nos problèmes ? Ce sont des problèmes d'ordre religieux, psychologique, économique, social qui se présentent à nous comme pratiquement insolubles. Et ils nous apparaissent tels parce que nous ne les comprenons pas. Et comment les comprendre ? Comment l'historien peut-il aider, lui, à les comprendre ? Par une connaissance élaborée de leurs origines, de leur évolution dans les groupes humains qui nous ont précédés de près ou de loin. Voyons de plus près ces problèmes. Dans le domaine économique et social, il est urgent de reprendre le travail historique dans telle direction que l'histoire des prix et des revenus - qui n'a pas encore été tentée que je sache - Pas une histoire des prix et des revenus pour le plaisir de construire de la statistique. Ce ne sont pas les chiffres qui nous intéressent, mais les hommes. Et une histoire des prix orientée vers l'histoire des revenus, des salaires et des fortunes. Toute histoire économique sans une histoire des prix et des revenus préalablement établie, est précaire, à plus forte raison l'histoire sociale qui est la véritable histoire. Qu'on ne vienne pas dire que c'est là une explication matérialiste de l'histoire. C'est seulement un élément indispensable, et non pas le seul, à l'explication des phénomènes économiques et surtout 
sociaux. A ce moment, la critique statistique vient appuyer selon ses moyens la critique historique, comme l'a si bien démontré M. Ernest Labrousse 7 , l'histoire quantitative s'ajoute à l'histoire qualitative. Quand on aura fait l'histoire des prix et revenus de la période 1730-1820, par exemple, on pourra faire l'histoire économique et sociale de la même période, et on pourra voir à ce moment quels changements profonds se sont produits. L'histoire de la conjoncture et des fluctuations économiques qui fournira enfin des indications à l'analyse économique, mais qui servira surtout à l'histoire des groupes sociaux, des classes populaires et bourgeoises. - Et de grâce, n'allons pas confondre l'histoire économique avec la seule histoire monétaire et encore moins avec la politique économique ou la législation économique.

Il faut encore expliquer les conséquences sociales des changements de structure que l'industrialisation a apportés depuis cent ans; changements de structure dont on ne fait que pressentir l'existence grâce à l'apport des romanciers et des sociologues. L'histoire de la colonisation au Canada, l'histoire agraire: pas une histoire de l'agriculture, mais une histoire de la campagne, du milieu rural, de l'appropriation du sol, de l'occupation de la terre, des modes et des types de culture, de la vie quotidienne à la campagne. Histoire des groupes sociaux urbains, histoire du mouvement ouvrier, qui ne se confond pas nécessairement avec l'histoire du syndicalisme, histoire des classes bourgeoises.

Passons à l'histoire religieuse. Il est important de reprendre notre histoire religieuse, d'en regrouper les divers éléments: les milieux d'importation française ou les influences originelles, les débuts héroïques, le lent développement et puis l'essor prodigieux de cette Eglise canadienne. Considérons encore les aspects missionnaire et confessionnel de cette religion, les milieux cléricaux, les rivalités entre clergé séculier et régulier, et certes oui les relations entre l'Eglise et l'Etat, plus particulièrement aux périodes de crise, comme nous l'a si bien montré un André Latreille dans son ouvrage sur l'Eglise pendant la Révolution fran-

7 Ernest Labrousse, Esquisse du mouvement des prix et des revenus en France au XVIIIe s. (2 vols., Paris, Dalloz, 1933). 
çaise. ${ }^{8}$ Et mettons-nous en frais de construire parallèlement l'histoire du sentiment religieux de la société canadienne par l'étude systématique de toute la « littérature religieuse »: je veux dire les prônes, les sermons, la prédication sous toutes ses formes. Cherchons encore dans l'enseignement donné dans les grands et petits séminaires, dans la vie religieuse du peuple, dans sa façon de pratiquer ses dévotions, son culte, dans ses réactions devant les grandes étapes de la vie. Dans ce sens, il est beaucoup plus important de connaître les rites de la mort que la liste des évêques, des curés et de leurs bedeaux. Cette histoire du sentiment religieux ne manquerait pas d'éclairer le comportement des Canadiens à un moment où cet aspect de leur vie est si controversé. Compréhension du passé... Peut-être pourrions-nous voir ensuite plus clairement dans ce qu'on appelle notre «jansénisme » ou notre «puritanisme ».

Du côté de l'histoire psychologique, comment comprendre notre mentalité de refoulés, de casaniers, de xénophobes ? N'aurait-elle pas une explication partielle dans le changement de vie qui s'opéra chez le groupe canadien de 1760-1800 ? Ces hommes, qui avaient été pendant cent ans des coureurs de bois par goût, des soldats par obligation et pour tout dire des aventuriers de grande classe, ces hommes sont devenus des cultivateurs rivés à l'entretien de leurs champs entourés de clôtures de cèdre et devant se replier entièrement vers les douceurs de la vie de famille. ${ }^{9}$ - Cette histoire économique, cette histoire sociale et psychologique doit être entreprise par des historiens de métier et avec l'étroite collaboration des sociologues et des économistes.

Histoire des idées, la plus belle qui soit. Je pense ici à l'histoire des influences, européennes, françaises ou anglaises et américaines. Montesquieu, Rousseau et les Encyclopédistes ont eu une audience qu'on n'a pas encore bien évaluée, chez les intellectuels canadiens-français du dix-neuvième siècle. Je pense

${ }^{8}$ André Latreille, L'Eglise catholique et la Révolution française (2 vols., Paris, Hachette, 1946 et 1950$)$.

9 Edmond De Nevers, L'Avenir du peuple canadien-français (Paris, Henri Jouve, 1896), 42-43. 
encore aux Veuillot et aux Maurras qui ont été pratiqués quotidiennement par plusieurs générations de lecteurs de journaux et de revues. Une immense portion de l'opinion publique juge encore de tout et de tous à travers le prisme déformant du veuillotisme, où la France depuis 1789 est considérée comme un pays de perdition. Le maurrassisme a plongé des racines extrêmement profondes, ayant trouvé, il est vrai, un terrain parfois assez favorable chez le Canadien français. - Histoire de l'enseignement, histoire des arts, histoire des techniques matérielles, immenses chantiers qui attendent leurs maîtres d'œuvre et leurs ouvriers.

Que deviennent parmi tout cela les vieilles catégories, celles de l'histoire politique, diplomatique, militaire ? Il ne s'agit pas de les abandonner pour autant. Au contraire, il faut continuer, mais au lieu de s'en tenir à l'histoire de la politique royale ou à celle des sessions parlementaires, on devra s'attacher à étudier les institutions. Il est par exemple très important de bien comprendre la transplantation du régime parlementaire anglais du dix-huitième siècle, avec son patronage et sa corruption électorale, avec ses factions et son absence de partis politiques, état de choses qui n'est pas totalement disparu. Une telle histoire serait de nature à expliquer notre mentalité politique essentiellement vénale et notre absence d'idées et de doctrines sur le plan politique.

Histoire problématique donc, qui doit partir de problèmes à élucider. Et comme en mathématiques, il importe de poser des hypothèses avant de procéder. ${ }^{10} \mathrm{~A}$ ce niveau, l'histoire n'est plus du donné mais du construit. - Organisation du passé en fonction du présent; c'est la fonction sociale de l'histoire, Marc Bloch affirme que l'ignorance du passé ne se borne pas à nuire à la connaissance du présent mais qu'elle compromet dans le présent l'action même. Entendue ainsi, l'histoire doit tôt ou tard nous aider à mieux vivre. Est-il besoin d'ajouter qu'il y a une différence essentielle entre l'histoire recherchée comme justification du présent, et l'histoire construite en vue de comprendre le passé, pour mieux comprendre le présent.

10 Lucien Febvre, Combats pour l'histoire (Paris, Colin 1953), 22-23. 
Ces nouvelles séries vont bouleverser quelque peu notre notion habituelle du document. Avec quels documents doit-on écrire cette histoire ? Avec les documents d'archives, et pas seulement la correspondance des séries coloniales ou diplomatiques, mais encore avec ces archives d'une richesse inouie que sont les minutes de notaire et les archives judiciaires en général. Avec les documents imprimés de toute nature. Est-ce tout? S'il en était ainsi, la vie se confondrait avec l'écriture, ce qui n'est pas le cas. L'histoire doit s'appuyer sur n'importe quel témoignage, qu'il soit d'ordre géologique, botanique, statistique, démographique, folklorique, iconographique, bref sur toute trace laissée par les hommes, les collectivités. ${ }^{11}$

Considérée dans cette perspective, la recherche en histoire devient extrêmement exigeante pour l'historien, pour les maîtres d'œuvre sinon pour les bons ouvriers. Elle exige au premier chef une forte culture générale dans les sciences humaines. En histoire d'abord, bien entendu; histoire du Canada, histoire de l'Amérique, histoire de l'Europe. Incidemment, il ne faudrait pas oublier que nulle histoire du Canada ou de la province de Québec n'est possible sans une connaissance approfondie de l'histoire de la France, de l'Angleterre, de l'Empire britannique ou du Commonwealth, selon les moments de notre histoire. Connaissance de l'histoire appuyée sur la critique historique la plus rigoureuse, bien entendu. Connaissances en géographie ensuite, en économique, en sociologie, en démographie, en histoire de l'art. Et encore. Il ne s'agit pas de tout savoir de ces disciplines, mais bien de leur emprunter une méthode et un esprit. Alliance des méthodes qui appelle la collaboration des hommes. On doit organiser la connaissance du passé sur le même plan que la connaissance du présent, sur le plan de l'équipe; équipe d'historiens sous la direction d'un maître, oui, mais aussi équipe d'historiens, de sociologues et d'économistes. Equipes qui travailleront par monographies régionales. On arrivera peut-être ainsi un jour à l'étude globale des ensembles historiques. En tous cas, cette histoire a beaucoup plus de chance de nous montrer l'unité profonde du travail scientifique et de parvenir au terme ultime

11 Lucien Febvre, Combats pour l'histoire, 13. 
de la recherche: l'historien n'est pas celui qui sait mais celui qui comprend. J'allais oublier une exigence préalable: celle de l'appréhension du vivant. L'intérêt pour le présent est d'importance première, et comme disait Pirenne, l'historien c'est celui qui aime la vie et qui sait la regarder. ${ }^{12}$

«Dans notre connaissance de l'homme, a écrit Charles Morazé, nous découvrons au hasard des trous énormes qui bloquent tout progrès et dont, bien évidemment, les spécialistes ne peuvent avoir conscience que par hasard, sur les bords de leur spécialité, et sans jamais en mesurer l'ampleur.... ${ }^{13}$ Est-ce qu'il n'en serait pas ainsi de l'Histoire du Canada actuellement ? $\mathrm{Ne}$ serions-nous pas en train de tourner en rond, de passer à côté des vrais problèmes de la recherche historique, faute de nous arrêter et de réfléchir?

$\mathrm{Au}$ terme de ce trop court exposé, je ne crains pas d'affirmer que l'histoire est une discipline scientifique dont l'objet n'est pas le fait, l'événement, mais bien l'homme, les hommes vivant en groupes. Que la compréhension de ces sociétés humaines d'autrefois exigent non seulement qu'on reprenne l'étude de l'histoire traditionnelle, mais encore qu'on entreprenne des recherches dans des directions jusqu'ici ignorées, qu'on étudie de nouvelles séries de phénomènes qui correspondent aux préoccupations des sociétés actuelles. Et puisqu'il faut faire un choix parmi les faits humains, il convient d'étudier les phénomènes à long terme, tels que le sentiment religieux, les classes sociales ou les institutions politiques, et négliger les phénomènes à court terme, tels que certains événements politiques ou militaires de médiocre importance ou de faible amplitude. En un mot, chercher le permanent surtout s'il nous paraît banal et évident. ${ }^{14} \mathrm{Je}$ n'hésite pas non plus à redire qu'au niveau supé-

12 Henri Pirenne, cité en Febvre, Combats pour l'histoire, 32.

13 Charles Morazé, Trois essais sur histoire et culture, Cahiers des Annales, no 2, Colin (1949), 62.

14 Jean Fourastié, in Rapports du IXe congrès international des sciences historiques (Colin, 1950), 217. 
rieur de la recherche, l'histoire ne doit jamais être mise au service d'un parti ou d'un système, qu'elle n'est pas un trésor où l'on puise des justifications pour l'engagement immédiat. Si l'histoire est remise en cause par tant de gens et tant d'opinions, ayons le courage de repenser notre discipline. Seule cette démarche de l'esprit effectuée par les hommes du métier justifiera la jeunesse de Clio.

Claude GalarnEaU

OUVRAGES REÇUS et dont un compte rendu paraîtra dans le prochain numéro de la Revue:

$1^{\circ}$. Léon Pouliot, s.j., Monseigneur Bourget et son temps. Tome I - Les années de préparation (Éditions Beauchemin, Montréal, 1955). 210 pages.

Nous recommandons très particulièrement cet ouvrage de l'un de nos directeurs. On sait que le Père Pouliot est un maître.

$2^{\circ}$. Gustave Lanctot, Une Nouvelle-France inconnue (Montréal, Librairie Ducharme, 1955). 205 pages.

$3^{\circ}$. Roland-J. Auger, La Grande Recrue de 1653 (Publications de la Société Généalogique canadienne-française, no 1, Montréal, 1955). 207 pages.

$4^{\circ}$. Robert-Lionel SÉGUIN, Le Mouvement insurrectionnel dans la Presqu'̂́le de Vaudreuil 1837-1838 (Librairie Ducharme, Montréal, 1955). 158 pages.

$5^{\circ}$. Rossel VIEN, Histoire de Roberval, cour du Lac-Saint-Jean. 1855-1955 (Publications de la Société historique du Saguenay, 1955). 369 pages.

$6^{\circ}$. Louis C. de LÉRY, s.j. Le problème du divorce (Les Éditions Bellarmin, Montréal, 1955). 137 pages.

$7^{\circ}$. Joseph et Viateur DuRAND, c.s.v. Jean Durand et sa postérité (Les Clercs Saint-Viateur, Outremont, 1954). 460 pages. 